\title{
NPR3 inhibits EMT and Tumour Metastasis of Human Osteosarcoma by Activating AKT/mTOR- mediated Autophagy
}

\section{Zhihong Yao}

Yunnan Cancer Hospital

\section{Yihao Yang}

Yunnan Cancer Hospital

Jiaxiang Chen

Yunnan Cancer Hospital

Ting Chen

Yunnan Cancer Hospital

Lei Han

Yunnan Cancer Hospital

Dongqi Li

Yunnan Cancer Hospital

Lijuan Ye

Yunnan Cancer Hospital

Yanan Zhu

Yunnan Cancer Hospital

Yan Feng

Yunnan Cancer Hospital

Jia Wu

Yunnan Cancer Hospital

Xinchao Yu

Yunnan Cancer Hospital

Zuozhang Yang ( $\nabla$ yangzuozhang@kmmu.edu.cn )

Yunnan Cancer Hospital

\section{Research}

Keywords: NPR3, osteosarcoma, metastasis, EMT, autophagy, apoptosis

Posted Date: September 28th, 2021

DOl: https://doi.org/10.21203/rs.3.rs-923877/v1 
License: (c) (i) This work is licensed under a Creative Commons Attribution 4.0 International License. Read Full License 


\section{Abstract}

\section{Background}

Osteosarcoma is an aggressive primary malignant cancer of bone mainly occurring in adolescence with a characteristic of high metastasis and relapse rate. In our previous study, we first identified that NPR3 was significantly decreased in OS samples. Here, we purposed to investigate the effect and the possible mechanisms of NPR3 on the progression of human OS.

\section{Methods}

The expression of NPR3 in OS patients and cells was detected by qRT-PCR, and IHC analysis. The effect of the expression of NPR3 on tumour metastasis was examined in vitro and in vivo. The molecular mechanisms of the regulation of NPR3 were evaluated in vitro and in vivo. The clinical relevance of 5year overall survival with the expression of NPR3 was evaluated in 294 patients with OS.

\section{Results}

Firstly, we indicated that NPR3 was substantially downregulated expression in OS tissues and cells by qRT-PCR and IHC assay. And the patients with lower expression of NPR3 have a poor prognosis. Functional studies revealed that over-expression of NPR3 inhibited the proliferation and invasion of cells. Meanwhile, over-expression of NPR3 markedly inhibited tumorigenesis and weakened tumour metastasis in vivo. Interestingly, we found that over-expression of NPR3 could induce autophagy, promote apoptosis and inhibit EMT. Additionally, overexpression of NPR3 decreased the phosphorylation levels of AKT and mTOR. Loss-of-function experiments displayed that effects of NPR3 were weakened by treatment with the specific autophagy inhibitor Baf-A1 and CQ.

\section{Conclusions}

Taken together, these results demonstrated that down-regulation of NPR3 promote lung metastasis of human OS by promoting EMT in part through the AKT/mTOR mediated autophagy, suggesting that NPR3 has therapeutic potential for OS patients with metastasis.

\section{Background}

Osteosarcoma (OS) is an aggressive primary malignant cancer of bone mainly occurring in adolescence with the characteristic of high metastasis and relapse rate compared with other bone tumors [1]. In clinical, those patients were often diagnosed in an advanced stage, and almost $90 \%$ of patients died from pulmonary metastases[2]. Despite the use of the neoadjuvant chemotherapy, the 5-year survival of OS with metastatic disease is approximately $20 \%[3,4]$. Currently, the overall clinical prognosis of patients with OS is poor, moreover there are no effective targeted agents approved for OS. Hence, there is an urgent need to explore the new key protein biomarkers for metastatic or relapsed OS to improve their prognosis. 
The mechanisms of OS development are complex, but some progress has been made. Efforts in the past few decades have focused on the mechanism of some non-coding RNAs (ncRNAs) [5], autophagy [6] and EMT pathways [7] in the metastasis of OS, as well as in sporadic OS. It is noteworthy that some potential target genes are mainly concentrated in the PI3K/Akt/mTOR pathway, making PI3K/AKT/mTOR pathway identified as potentially valuable therapeutic target exploitation. Coincidentally, small molecule inhibitors for this pathway have shown encouraging results [8]. In our previous study, we identified a differentially low expressed target gene NPR3 in OS patients using meta-analysis for the first time [9]. However, the clinical value of NPR3 in OS patients with metastasis remains elucidated. NPR3, located at human chromosome 16p13.3, is a member of the natriuretic peptide receptor family expressed in numerous normal tissues, including the skeletal muscle, heart, kidney, liver, and pancreas [10]. Accumulated research reported that NPR3 was play critical roles in cartilage homeostasis, preserves cardiac function [11-13]. The latest study mentions its role in colorectal cancer (CRC) [14] and clear cell renal cell carcinoma (ccRCC) metastasis [15]. Intriguingly, advanced studies have provided the evidence proving the relationship between NPR3 and PI3K/AKT pathway, autophagy pathway $[16,17]$. Thus, it is very essential to explore the regulatory mechanisms of this connection in tumorigenesis and progression of human OS.

In this paper, we first explored the function role of NPR3 in the development of OS. Furthermore, we intend to explored whether NPR3 affects EMT and autophagy of OS through the AKT/mTOR pathway. This study is expected to provide a new idea for the treatment of OS.

\section{Materials And Methods}

\section{Patients and clinical specimens}

In this study, a total of 122 fresh primary bone tumour tissues with metastases and 172 bone tumour tissues without distance tissues metastases of OS patients who underwent surgery were collected from Yunnan Cancer Hospital, Kunming, China (December 2013 to December 2020). These tissues were stored at $-80^{\circ} \mathrm{C}$ immediately after surgery. All the resected tissues were identified by pathologic examination. RNA level of NPR3 was measured by those samples. All samples were staged according to Enneking surgical staging [18]. The median fellow-up time was 22 months. Overall survival was defined as the interval between the date of surgery and death or the end point of follow up. Paraffin sections of $19 \mathrm{OS}$ cases with and without lung metastases were obtained from the Department of Pathology of the Yunnan Cancer Hospital, Kunming, China (May 2019 to December 2020) including the primary bone tumour tissues, para-cancer tissues. Protein levels of NPR3 was measured by those paraffin sections. Written informed consent was obtained from all patients. This study was approved by the Ethics Committee of Yunnan Cancer Hospital.

\section{Cell culture and transfection}

Human normal osteoblastic cell hFOB, and immortalized OS cell lines U2OS, HOS-mnng were purchased from Shanghai Institutes for Biological Sciences, Chinese Academy of Science (Shanghai, China). And 
the MG63 and Saos-2 cells were obtained from the Cell Resource Centre of Kunming institute of Zoology, Chinese Academy of Science (Kunming, China). All cell lines were cultured in Dulbecco's Modified Eagle Medium (DMEM, Gibco, CA) with $10 \%$ fetal bovine serum (FBS) and $1 \%$ streptomycin/penicillin at $37^{\circ} \mathrm{C}$, $5 \% \mathrm{CO}_{2}$. The plasmids pcDNA3.1-EGFP-NPR3 and pcDNA-EGFP-NC were purchased from the Honor-Gene company to overexpress NPR3. The cells stably over-expressing (OE) of NPR3 was constructed in our lab. The transfection experiments were performed according to the manufacturer's instructions included with Lipofectamine 2000 TM (Invitrogen). Stable cell lines were cultured in complete medium supplemented with $600 \mu \mathrm{g} / \mathrm{ml} \mathrm{G} 418$.

\section{Immunocytochemistry analysis (IHC)}

A total of 57 paraffin-embedded OS primary bone tumour samples from 19 patients with OS were collected from the Department of Pathology, Yunnan Cancer Hospital. After that, the slides were incubated with 1:500 dilution of antibody to NPR3 (Abcam) at $4^{\circ} \mathrm{C}$ overnight and rinsed with phosphatebuffered saline (PBS). Then goat anti-rabbit secondary antibody labeled polymer-HRP was incubated for $1 \mathrm{~h}$ in dark. After wishing with PBS, the color reaction was developed by $3,3^{\prime}-$

diaminobenzidinetetrachloride (DAB), followed by counterstained with hematoxylin. The negative control is the IgG isotype antibody. Samples were examined with a fluorescence microscope (Nikon). Then we scored the staining intensity by two independent pathologists. The IHC scored divided into 4 grades as follows: 0 (negative, < 5\%), 1 (weak, 5-25\%), 2 (moderate, 25\%-50\%), 3 (strong, 50\%-75\%), 4(strong, > $75 \%) . p$ values were based on the two-sided statistical analysis and $p$-value less than 0.05 was considered to be statistically significant.

\section{qRT-PCR}

Total RNA was extracted from cultured OS cell lines or specimens using the TRIzol reagent (Promega). The reverse transcription and the qRT-PCR were carried out as previously described [19]. The total RNA was reverse-transcribed using a First Strand cDNA Synthesis Kit (Promega). qRT-PCR was performed using the SYBR qPCR Master Mix (Promega). Expression data were normalized to the geometric mean of the housekeeping gene GAPDH to control the variability in expression levels and calculated using the $2^{-\Delta \Delta C t}$ method. $\Delta \Delta \mathrm{Ct}=$ [Ct (gene of interest, sample)-Ct (GAPDH, sample)]- [Ct (gene of interest, calibrator)-Ct (GAPDH, calibrator)].

The primers of NPR3 was used for qPCR as follows, 5-ACCCTGGACCGTATACCCTGA-3 (forward), 5CACCACTTTCTCGTTCTGGCC-3 (reverse).

\section{Western Blotting (WB)}

This assay was performed as previously described [20]. Proteins were extracted with cold lysis buffer. Following centrifugation, the supernatant was harvested. After rationing by the BCA protein concentration assay kit, the protein samples were electrophoresed on $12 \%$ SDS-PAGE, and transferred onto PVDF membrane. After blocking with $5 \%$ nonfat milk in tris-buffered saline buffer, the membranes were incubated with primary antibodies at $4^{\circ} \mathrm{C}$ overnight, and reacted with horseradish peroxidase-conjugated 
secondary antibodies. Blot bands were developed by enhanced chemiluminescence reagents. In this research, the antibodies were as follows: NPR3 (ab177954, Abcam), p62 (ab207305, Abcam), LC3 $8(a b 52628$, Abcam), LC3区(ab192890, Abcam), p-mTOR (ab278621, Abcam), mTOR (ab134903, Abcam), p-AKT (ab38449, Abcam), t-AKT (ab207452, Abcam), E-cadherin (ab1416, Abcam), Ncadherin(ab76011, Abcam), vimentin (ab92547, Abcam), snail (ab216347, Abcam), twist 1 (ab50887, Abcam), ZEB1 (ab203829, Abcam), $\beta$-actin (ab8226, Abcam).

\section{Wound healing assay}

Both non-transfected and stably-transfected MG63 and Saos-2 cells were cultured in 12-well plates respectively, media was changed to $0.1 \%$ FBS DMEM before scratching. Scratch wound was generated using a $10 \mu \mathrm{l}$ micropipette tip after $12 \mathrm{~h}$, and then photographs were captured at indicated time points for $0,12 \mathrm{~h}$. The percentage of wound healing was calculated using the Image $\mathrm{J}$ software. Wound healing rate $(\%)=$ (initial wound area - non-healing area)/initial wound area.

\section{Matrigel Invasion Assay}

The assay was carried out using the BD Bio-coat Matrigel Invasion Chamber with inserts of 8- $\mu \mathrm{m}$ pore size for 24-well plates (BD Biosciences) as the manufacturer's instructions. Briefly, the OS cells were suspended with medium without FBS, then $5 \times 10^{4}$ cells were plated in the upper chamber in 200ul serumfree medium. The bottom chamber contained complete medium with $10 \% \mathrm{FBS}$. Undergoing migration for $12 \mathrm{~h}$, the cells that had invaded through the membrane to the lower surface were fixed with $4 \%$ paraformaldehyde and stained with $0.1 \%$ Crystal violet for $1 \mathrm{~h}$ at room temperature. The cell numbers were counted in five random fields of each chamber under the microscope (Nikon).

\section{Cell viability assays}

Cell proliferation was measured by the Cell Counting Kit-8 (CCK-8) assay following the manufacturer's instructions. Briefly, the cells at a density of $5 \times 10^{3}$ cells / well were cultured in 96 -well plates. The mixture was then treated with CCK-8 reagent. The absorbance at $450 \mathrm{~nm}$ was measured to determine the cell proliferation viability.

\section{Cell apoptosis assay}

To investigate the effect of NPR3 on cell apoptosis, apoptosis was evaluated by flow cytometry (FC) using the annexin V apoptosis detection FITC Kit. Data obtained from early and late apoptosis are depicted together as apoptosis in the results. The apoptotic effect on OS cells was confirmed by Annexin V-FITC/PI double staining. Statistical analysis was performed using the Mann-Whitney U test.

\section{Xenograft tumour model}

To evaluate the effect of NPR3 on primary tumour growth and spontaneous metastasis, we do the animal model. The male BALB/C nude mice (4 weeks old, purchased from Beijing Vital River Ltd., China) were injected with $2 \times 10^{6} / 50 \mu \mathrm{L}$ MG63-OE-NPR3 MG63 cells or OE-NC MG63 cells in tibial medullary cavity in each group. One week later, mice were divided into different groups $(n=15)$ and injected with normal 
saline or Baf-A1 ( $75 \mathrm{~nm} / \mathrm{kg} / \mathrm{d})$ every day for 3 weeks. Tumour volume and weight were measured beginning 5 weeks post injection and measured every 2 days until killing at 8 weeks. On day 56 , the IVIS Lumina Imaging System (Xenogen) was used to capture the signal by taking photographs. The tumours were excised, weighed, photographed, and processed for WB, IHC assays. Lungs, livers, kidney were processed for routine HE staining. And, the number of metastatic nodules in the lung was quantified. All experimental procedures were approved by the animal care and use committee of Kunming Medical University.

\section{Statistical analysis}

All statistical analyses were carried out using GraphPad 7.0 software (San Diego, CA) and SPSS 25.0 (Chicago, IL, USA). All results were reported as mean \pm SD from at least three separate experiments. The associations between NPR3 expression and the clinicopathological characteristics of the patients were analyzed using the Chi-squared test. Statistical analysis between different groups were performed with one-way ANOVA, between two groups were analyzed by Student's t test. The overall Survival curves were plotted using the Kaplan-Meier method and compared using the log-rank test. $p$ values $<0.05$ was considered statistically significant differences.

\section{Results}

\section{NPR3 was significantly down-regulated in metastatic human OS patents}

Our previous work identified a differentially down-regulated expression protein NPR3 in OS patients using meta-analysis for the first time [9]. To better understand the effect of NPR3 on the metastasis of OS, we examined the protein and mRNA levels of NPR3 in OS tissues with and without distance metastases. Interestingly, we observed that NPR3 was dramatically lower expression in tissues with metastases than in tissues without metastases at both the mRNA level and protein level (Fig. 1a, 1b,1d). The associated AUC in the ROC analysis was as high as $0.840(0.7951-0.8844, p<0.001)$ (Fig. 1C). Moreover, we found that NPR3 was down-regulated expression in MG63 and Saos-2 than in the two other OS cell lines and 1 normal bone cell lines by qRT-PCR assay (Fig. 1e). Therefore, we selected the MG63 and Saos-2 to carry out the subsequent experiments in this research. These results suggested that NPR3 may promote the metastasis of OS.

\section{Down-regulated expression of NPR3 was correlated with poor prognosis of OS patients}

The correlation between NPR3 express and clinicopathologic characteristic was shown in Table 1. The analysis of the relationship between NPR3 expression level and clinical features of OS patients was shown in Table 2. We found that the expression level of NPR3was correlated with clinical stage $(p=$ $0.016)$, tumour metastasis $(p<0.001)$ and tumour recurrence $(p=0.002)$. No difference was found for 
age, gender, tumour size, BMI, primary tumour site or pathological fracture. The risk factors associated with the overall survival of OS patients were shown in Table 3. Moreover, Kaplan-Meier survival analysis indicated that the clinical stage, metastasis and NPR3 expression level were the independent prognosis factor for the overall survival of OS. And the patients with low level of NPR3 had poorer overall survival than those with high expression level of NPR3 $(p<0.001)$ (Fig. 1f). Together, these results strongly suggested the NPR3 was significantly correlated with the prognosis of OS. 
Table 1

Correlation between NPR3 expression and Clinicopathologic characteristics in 294 patients with osteosarcoma

\begin{tabular}{|c|c|c|c|c|}
\hline \multirow[t]{2}{*}{ Characteristics } & \multirow[t]{2}{*}{ Number of cases (\%) } & \multicolumn{2}{|l|}{ NPR3 expression } & \multirow[t]{2}{*}{$p$} \\
\hline & & High no. cases (\%) & Low no. cases (\%) & \\
\hline Age, y & & & & 0.579 \\
\hline$\leq 35$ & 261 (88.78) & $129(87.76)$ & $132(89.80)$ & \\
\hline \35 & $33(11.22)$ & $18(12.24)$ & $15(10.20)$ & \\
\hline Gender & & & & 0.803 \\
\hline Male & $200(68.03)$ & $101(68.71)$ & 99 (67.35) & \\
\hline Female & $94(31.97)$ & $46(31.29)$ & 48 (32.65) & \\
\hline Tumour size, cm & & & & 1 \\
\hline$\otimes 10$ & $144(48.98)$ & $72(48.98)$ & $72(48.98)$ & \\
\hline$\geq 10$ & $150(51.02)$ & 75 (51.02) & 75 (51.02) & \\
\hline BMI & & & & 0.416 \\
\hline$\varangle 22$ & $222(75.51)$ & $108(73.47)$ & 114 (77.55) & \\
\hline$\geq 22$ & $72(24.49)$ & $39(26.53)$ & $33(22.45)$ & \\
\hline Clinical stage & & & & 0.395 \\
\hline$\| \mathrm{A}$ & $43(14.63)$ & $22(14.97)$ & 21 (14.29) & \\
\hline IIB & $211(71.77)$ & 109 (74.15) & $102(69.39)$ & \\
\hline III-IV & $40(13.60)$ & $16(10.88)$ & $24(16.32)$ & \\
\hline Primary tumour site & & & & 0.804 \\
\hline Femur & $149(50.69)$ & $74(50.35)$ & 75 (51.03) & \\
\hline Fibula & $19(6.46)$ & $11(7.48)$ & $8(5.44)$ & \\
\hline Humerus & $15(5.10)$ & $8(5.44)$ & $7(4.76)$ & \\
\hline Tibia & 89 (30.27) & $46(31.29)$ & $43(29.25)$ & \\
\hline Pelvis & $6(2.04)$ & $2(1.36)$ & $4(2.72)$ & \\
\hline Other* & $16(5.44)$ & $6(4.08)$ & $10(6.80)$ & \\
\hline Pathological fracture & & & & 0.589 \\
\hline
\end{tabular}

*other: including ilium, metacarpal bone, patella, radial, ribs, vertebral, scapula. 


\begin{tabular}{|c|c|c|c|c|}
\hline \multirow[t]{2}{*}{ Characteristics } & \multirow[t]{2}{*}{ Number of cases (\%) } & \multicolumn{2}{|l|}{ NPR3 expression } & \multirow[t]{2}{*}{$p$} \\
\hline & & High no. cases (\%) & Low no. cases (\%) & \\
\hline Yes & $35(11.90)$ & $16(10.88)$ & $19(12.93)$ & \\
\hline No & $259(88.10)$ & $131(89.12)$ & $128(87.07)$ & \\
\hline Metastasis & & & & $<0.001$ \\
\hline Yes & $122(41.50)$ & $25(17.01)$ & $97(65.99)$ & \\
\hline No & $172(58.50)$ & $122(82.99)$ & $50(34.01)$ & \\
\hline Recurrence & & & & 0.076 \\
\hline Yes & $22(7.48)$ & $7(4.76)$ & $15(10.20)$ & \\
\hline No & $272(92.52)$ & $140(95.24)$ & $132(89.80)$ & \\
\hline
\end{tabular}

Table 2

Spearman analysis of correlation between NPR3 and Clinicopathological

\begin{tabular}{|lll|}
\hline Variables & NPR3 expression & \\
\cline { 2 - 3 } & Spearman Correlation & $\boldsymbol{p}$ \\
\hline Age, y & -0.013 & 0.826 \\
\hline Gender & -0.023 & 0.696 \\
\hline Tumour size, cm & 0.141 & 0.492 \\
\hline BMI & 0.020 & 0.731 \\
Clinical stage & -0.140 & 0.016 \\
\hline Primary tumour site & -0.075 & 0.198 \\
\hline Pathological fracture & 0.007 & 0.903 \\
\hline Metastasis & -0.580 & $<0.001$ \\
\hline Recurrence & -0.180 & 0.002 \\
\hline
\end{tabular}


Table 3

Univariate and multivariate Cox proportional hazards regression analysis for the risk factors associated with the survival of OS patients

\begin{tabular}{|c|c|c|c|c|}
\hline \multirow[t]{2}{*}{ Variables } & \multicolumn{2}{|c|}{ Univariate analysis } & \multicolumn{2}{|l|}{ Multivariate analysis } \\
\hline & $\mathrm{HR}(95 \% \mathrm{Cl})$ & $p$ & $\mathrm{HR}(95 \% \mathrm{Cl})$ & $p$ \\
\hline Age, y & $\begin{array}{l}1.001(0.986- \\
1.016)\end{array}$ & 0.895 & $0.992(0.497-1.978)$ & 0.981 \\
\hline Gender (male vs female) & $\begin{array}{l}0.783(0.518- \\
1.185)\end{array}$ & 0.248 & $0.816(0.528-1.263)$ & 0.362 \\
\hline Tumour Size (< 10 vs $\geq 10$ ) & $\begin{array}{l}1.060(0.731- \\
1.536)\end{array}$ & 0.760 & $0.955(0.644-1.416)$ & 0.819 \\
\hline $\mathrm{BMI}(<22$ vs $\geq 22)$ & $\begin{array}{l}1.042(0.676- \\
1.608)\end{array}$ & 0.851 & $0.890(0.545-1.453)$ & 0.642 \\
\hline Clinical stage (IIA vs IIB vs III) & $\begin{array}{l}1.984(1.412- \\
2.788)\end{array}$ & $<.001$ & $1.302(0.897-1.890)$ & 0.166 \\
\hline Primary tumour site & $\begin{array}{l}1.026(0.934- \\
1.128)\end{array}$ & 0.591 & $1.028(0.933-1.1330$ & 0.572 \\
\hline $\begin{array}{l}\text { Pathological fracture (yes vs } \\
\text { no) }\end{array}$ & $\begin{array}{l}0.954(0.524- \\
1.737)\end{array}$ & 0.878 & $0.973(0.510-1.845)$ & 0.926 \\
\hline Recurrence (yes vs no) & $\begin{array}{l}1.832(1.046- \\
3.209)\end{array}$ & 0.034 & $1.201(0.668-2.160)$ & 0.541 \\
\hline Metastasis (yes vs no) & $\begin{array}{l}5.237(3.421- \\
8.017)\end{array}$ & $<.001$ & $2.255(1.323-3.843)$ & 0.003 \\
\hline NPR3 expression (high vs low) & $\begin{array}{l}0.449(0.366- \\
0.552)\end{array}$ & $\stackrel{<}{0.001}$ & $0.543(0.429-0.688)$ & $<0.001$ \\
\hline
\end{tabular}

Abbreviations: HR, hazard ratio; $\mathrm{Cl}$, confidence interval.

\section{Overexpression NPR3 inhibited the proliferation, migration and invasion of MG63 and Saos-2}

Next, we evaluated whether NPR3 regulated the proliferation, migration and invasion of OS. We generated stably-transfected cells with overexpression NPR3 expression in MG63 and Saos-2, and subsequently detected by qRT-PCR and WB after 48h (Fig. 2a, b). Then the proliferative rate, the migration and invasive ability of OS cells were tested. The results showed that NPR3 overexpression dramatically suppressed the proliferation of in OS cell lines MG63 and Saos-2 (Fig. 2c, d). Moreover, in transwell and matrigel assays, the overexpression of NPR3 significantly decreased the migration and invasive cell numbers compared with the control group and OE-NC group (Fig. 2e, f, g, h). These results demonstrated that overexpression of NPR3 inhibited the proliferation, cell migration and invasion of OS cells in vitro. 


\section{Over-expression of NPR3 inhibited the tumour growth and metastasis of OS in vivo}

We next investigated the effect of NPR3 in vivo. Stably-transfected MG63 cells were injected subcutaneously into tibial medullary cavity (left and right) of nude mice. The animals were monitored for tumour growth and distance metastases from 5 weeks. We observed that lung metastases were found in all five mice (15/15) from the OE-NC group, whereas $66 \%$ nude mice were found having lung metastatic nodes in the OE-NPR3 group (10/15) (Fig. 3a). Compared to the control group, tumors were significantly smaller in volume and weight in OE-NPR3 group (Fig. 3b, c, d, e). The lung, liver and kidney tissue were collected to determine the effects of NPR3 on tumour metastasis (Fig. 3f). The results revealed that the degree of distant tissue metastasis was significantly higher in the OE-NPR3 group than in the OE-NC group $(p<0.001)$. These results confirmed the role of NPR3 in inhibiting tumour growth and distant metastasis in vivo.

\section{Over-expression of NPR3 induced cell death and inhibited EMT in OS cells}

Generally, metastasis is mainly carried out in four stages: cell survival, passing through the matrix into the circulatory system, colonizing into the distant metastatic sit, and activating. In this process, tumour cells often undergo EMT, then invade into the circulatory system [21]. Thus, we examined the effect of NPR3 on EMT and cell death. The WB assay revealed that overexpression of NPR3 in MG63 cells significantly the increased the expression level of epithelial cell marker E-cadherin, but decreased the expression levels of EMT-related proteins including N-cadherin, Vimentin, Snail, Twist1 and ZEB1 (Fig. 4a, b). Moreover, overexpression of NPR3 increased the expression level of cell apoptosis proteins Bax and caspase 3 and decreased the expression of Bcl-2 (Fig. 4c, d). Cell death of Saos-2 and MG63 cells was determined by staining with Annexin V-FITC and PI. Flow cytometry data confirmed that overexpression of NPR3 induced cell apoptosis and necrosis (Fig. 4e, f). These data above suggested that overexpression of NPR3 inhibited the metastasis of OS through inducing the cell death and suppressing the EMT.

\section{Effect of NPR3 on cell apoptosis and EMT may depends on the cell autophagy}

Previous studies reported autophagy effects on cell apoptosis [22]. Therefore, we assessed the possible association between NPR3 and cell autophagy in OS cell lines. We first investigated whether NPR3 could induce the cell autophagy. We found that the percentage of GFP-LC3 punctapositive cells was significantly increased in the OE-NPR3 group compared with the control group $(p<0.05)$ (Fig. 5a, b). As expected, Overexpression of NPR3 increased the expression of LC3\ while decreased the expression of p62 (Fig. 5c). Furtherly, to further investigate the underlying molecular basis for NPR3-mediated autophagy, we found that overexpression of NPR3 reduced phosphorylation levels of AKT and mTOR 
while no significant effect on total protein levels of AKT and mTOR in MG63 and Saos-2 cells (Fig. 5d). Collectively, these results indicated that NPR3 may act as a potential autophagy promoter partially depend on the AKT/mTOR pathway.

Autophagy inhibitors Baf-A1 and CQ attenuated the inhibitory effect of NPR3 on tumour invasion in vitro, tumour growth and metastasis in vivo

Finally, loss-of-function experiments were performed to confirm the interaction between autophagy and NPR3 in OS cells. As shown in Fig. 6(a, b, c, d), wound-healing assay showed that autophagy inhibitors Baf-A1 and CQ reversed the inhibitory effect of OE-NPR3 on inhibiting tumour invasion. Stable OS cells overexpressing NPR3 were injected into nude mice. At the indicate times, pulmonary metastasis, BLI, Micro-CT assay and X-ray were used to measure the primary tumour growth and lung metastasis in nude mice deal with OE-NPR3 and OE-NPR3 + Baf-A1. The results showed that OE-NPR3 inhibited the tumour growth and distance metastasis in nude mice, while the autophagy inhibitor Baf-A1 reversed the function of NPR3 overexpression (Fig. 6e, f, g, h, i, j, k). These results confirmed that NPR3 inhibit the tumour growth and distance metastasis mainly depend on the autophagy pathway.

\section{Autophagy inhibitors Baf-A1 attenuated the effect of NPR3 on the EMT}

Also, autophagy inhibitors reversed inhibited-EMT effect of OE-NPR3 in MG63 cells (Fig. 7a, 7c) and in Saos-2 cell (Fig. 7b, 7d). These results confirmed that overexpression of NPR3 inhibited the EMT partly depended on the autophagy.

\section{Discussion}

OS is an aggressive cancer with the characteristic of high metastatic rate and high rate of relapse. It is important to comprehensive analysis the potential mechanism in the development of OS. In our previous study, we identified a differentially low expressed target gene NPR3 in OS patients using meta-analysis for the first time [9]. Interestingly, we identified that patients with downregulated of NPR3 have higher metastasis rates and poorer prognosis than those with high expression of NPR3. Then, we examined the biologic function of NPR3 on OS. We found that the proliferation and invasion abilities were inhibited in OE-NPR3 cells compared with the control. In vivo, overexpression of NPR3 decreases the incidence of metastasis. Excitingly, we elaborated that low expression of NPR3 can promote distance tissues metastasis of OS, mainly by inhibiting autophagy-induced apoptosis and EMT. And this process partly depended on the AKT/mTOR pathway. Those results indicated that NPR3 may be a potential tumour suppressor protein for OS.

The previous studies reported that NPR3 was play critical roles in cartilage homeostasis, and regulate blood pressure [23-26]. However, there are few reports on the role of NPR3 in the tumourigenesis. Emerging studies showed NPR3 possess anti-tumour activity in some cancers. NPR3 significantly 
inhibited the proliferation and apoptosis of colorectal cancer cells [14]. Li et al. reported that NPR3 promotes Clear cell renal cell carcinoma metastasis via activating p38-MAPK signaling [15]. We investigated the anti-tumour effect of NPR3 on human OS for the first time [9]. The results strongly indicated that low expression of NPR3 is likely to correlate with metastasize and poor survival. Meanwhile, our data demonstrates that down-regulation of NPR3 inhibited autophagy and apoptosis, promoted EMT partly through the PI3K/AKT/mTOR pathway.

EMT-like phenotype is the characteristic of tumors with metastatic ability, which often occurs in nonepithelial tumors. Kahlert et al proved that a mesenchymal transition process involved in the onset and progression of sarcomas. More evidence showed OS cells undergo the EMT process that associate with their metastatic ability [27-29]. Consistently, we found NPR3 overexpression led to increase the expression level of E-cadherin and decrease the expression level of EMT-activating proteins, such as Ncadherin, vimentin, snail, twist1, ZEB1 which increased migratory and invasive potential [30]. These results suggested that NPR3 regulated the invasion of OS depend on EMT.

Autophagy, as a cancer-suppressive function, could inhibit metastasis by selectively down-regulating critical transcription factors of EMT [31]. Also, Iml1p-Npr2p-Npr3p complexes may regulate the formation of autophagosomes [32], which was strongly impaired in NPR3 defective cells or Arabidopsis thaliana [33]. Therefore, we monitored the regulation of NPR3 on cell autophagy. The data confirmed that overexpression of NPR3 could induce autophagy, including increased GFP-LC3II dot number, promote LC3I changed to LC3II, reduced p62 level. PI3K/AKT/mTOR pathway may be a key modulator of autophagy, which plays a pivotal role in tumour metastasis [34]. The activate of AKT have the ability to promote protein synthesis and cell growth through activating mTOR [35]. And the mTOR has the function to promote cellular macromolecular synthesis and suppresses autophagy [36]. Many studies suggest that aberrant activation of the AKT/mTOR pathway is associated with human OS [37-39]. Addition studies have demonstrated that various signaling pathways involved in the progression of OS, including Wnt, $\mathrm{PI3K} / \mathrm{AKT}$, and TSC1-TSC2 [40, 41]. In consistent with this view [16], our present results also revealed that NPR3 exposure inactivated phosphorylation of mTOR/AKT and induced autophagy. We further used Baf-A1 and CQ to inhibit autophagy, and reversely verify the correlation between autophagy and NPR3. The autophagy inhibitor Baf-A1 and CQ blocked OE-NPR3-inhibited migration, invasion and EMT in OS. These results established that down-regulation of NPR3 induces migration/invasion and EMT in OS partly through the AKT/mTOR pathway.

\section{Conclusions}

In this study, we reported a novel target gene which is down-regulated in metastatic OS and associated with poor prognosis of patients with OS. Further functional studied showed that overexpression of NPR3 inhibited migration and invasion ability of OS in vitro and tumour distant tissue metastasis in vivo. Mechanistically, we found that NPR3 induced autophagy partly via AKT/mTOR pathway. Conclusively, NPR3 inhibited OS metastasis by suppressing EMT via the AKT/mTOR mediated autophagy. Therefore, Targeting NPR3 might represent a therapeutic and preventive target against OS. 


\section{Abbreviations}

OS: Osteosarcoma; NPR3: Natriuretic Peptide Receptor 3; ncRNAs: non-coding RNAs; NP: Natriuretic Peptide; CRC: colorectal cancer; ccRCC: clear cell renal cell carcinoma; TNM: tumour node metastasis; DMEM: Dulbecco's Modified Eagle Medium; FBS: fetal bovine serum; qRT-PCR: Quantitative real-time PCR; IHC: immunohistochemistry; SD: standard deviation; mTORC1: mTOR complex 1;

\section{Declarations}

\section{Ethics approval and consent to participate}

All human tissues experiments were approved by the Institutional Ethics Committee of the Third Affiliated Hospital of Kunming Medical University (Yunnan Cancer Hospital, Yunnan Cancer Center). Written informed consent form for the experimental studies was obtained from the patients or their guardians.

\section{Consent for publication}

Not applicable.

\section{Availability data and materials}

All data generated or analyzed during this study are included in this published article and its additional files. Additional datasets analyzed during the current study are available from the corresponding author on reasonable request.

\section{Competing interests}

The authors declare no conflict of interest.

\section{Funding}

This work was financially funded by the National Science Foundation of China (Nos. U1702283, 81972764, 81960488, 81960426), The China Postdoctoral Science Foundation (Nos. 2018M643798XB, 2018M643799XB), Postdoctoral Training Program of Yunnan Province, the Joint Special Funds for the Department of Science and Technology of Yunnan Province-Kunming Medical University (Nos. 2019FE001-152, 2019FE001-238, 2019FE001-074), the Scientific Research Projects of Internal Research Institutions of Medicine (Nos. 2017NS197, 2018NS069), the Medical Reserve Talents of Yunnan Province (Nos. D-201603, H-2019072, H-2018001), Training program for 100 Young and middle-aged Academic and technical backbones of Kunming Medical University, Ten Thousand Personnel Training Project of Yunnan Province.

\section{Author's contributions}


$\mathrm{ZHY}$ and $\mathrm{YHY}$ contributed equally to this study. ZHY and ZZY conceived and designed the study, ZHY and TC supervised the study. YHY, JXC, DQL, YNZ, and YF conducted the experiments. LJY, HL, JW and XCY analyzed the results. ZHY wrote the manuscript. All authors reviewed and approved the final manuscript.

\section{Acknowledgements}

Not applicable.

\section{References}

1. Serlo J, Tarkkanen M, Sampo M, Vettenranta K, Riikonen P, Helenius I. Incidence, treatment and survival of paediatric patients with bone sarcomas in Finland from 1991 to 2005. Acta paediatrica (Oslo, Norway : 1992). 2015;104:738-745.

2. Kager L, Zoubek A, Potschger U, Kastner U, Flege S, Kempf-Bielack B, et al. Primary metastatic osteosarcoma: presentation and outcome of patients treated on neoadjuvant Cooperative Osteosarcoma Study Group protocols. Journal of clinical oncology : official journal of the American Society of Clinical Oncology. 2003;21:2011-2018.

3. Bacci G, Rocca M, Salone M, Balladelli A, Ferrari S, Palmerini E, Forni C, Briccoli A. High grade osteosarcoma of the extremities with lung metastases at presentation: treatment with neoadjuvant chemotherapy and simultaneous resection of primary and metastatic lesions. Journal of surgical oncology. 2008;98:415-420.

4. Smeland S, Bielack SS, Whelan J, Bernstein M, Hogendoorn P, Krailo MD, et al. Survival and prognosis with osteosarcoma: outcomes in more than 2000 patients in the EURAMOS-1 (European and American Osteosarcoma Study) cohort. European journal of cancer (Oxford, England : 1990). 2019;109:36-50.

5. Dean DC, Shen S, Hornicek FJ, Duan Z. From genomics to metabolomics: emerging metastatic biomarkers in osteosarcoma. Cancer metastasis reviews. 2018;37:719-731.

6. Akin D, Wang SK, Habibzadegah-Tari P, Law B, Ostrov D, Li M, et al. A novel ATG4B antagonist inhibits autophagy and has a negative impact on osteosarcoma tumors. Autophagy. 2014;10:2021-2035.

7. Wang L. ELF1-activated FOXD3-AS1 promotes the migration, invasion and EMT of osteosarcoma cells via sponging miR-296-5p to upregulate ZCCHC3. Journal of bone oncology. 2021;26:100335.

8. Chawla SP, Staddon AP, Baker LH, Schuetze SM, Tolcher AW, D'Amato GZ, et al. Phase II study of the mammalian target of rapamycin inhibitor ridaforolimus in patients with advanced bone and soft tissue sarcomas. Journal of clinical oncology : official journal of the American Society of Clinical Oncology. 2012;30:78-84. 
9. Yang Z, Chen Y, Fu Y, Yang Y, Zhang Y, Chen Y, Li D. Meta-analysis of differentially expressed genes in osteosarcoma based on gene expression data. BMC medical genetics. 2014;15:80.

10. Anand-Srivastava MB. Natriuretic peptide receptor-C signaling and regulation. Peptides. 2005;26:1044-1059.

11. Peake NJ, Hobbs AJ, Pingguan-Murphy B, Salter DM, Berenbaum F, Chowdhury TT. Role of Ctype natriuretic peptide signalling in maintaining cartilage and bone function. Osteoarthritis and cartilage. 2014;22:1800-1807.

12. Moyes AJ, Chu SM, Aubdool AA, Dukinfield MS, Margulies KB, Bedi KC, Hodivala-Dilke K, Baliga RS, Hobbs AJ. C-type natriuretic peptide co-ordinates cardiac structure and function. European heart journal. 2019.

13. Saulnier PJ, Roussel R, Halimi JM, Lebrec J, Dardari D, Maimaitiming S, et al. Impact of natriuretic peptide clearance receptor (NPR3) gene variants on blood pressure in type 2 diabetes. Diabetes care. 2011;34:1199-1204.

14. Gu L, Lu L, Zhou D, Liu Z. Long Noncoding RNA BCYRN1 Promotes the Proliferation of Colorectal Cancer Cells via Up-Regulating NPR3 Expression. Cellular physiology and biochemistry : international journal of experimental cellular physiology, biochemistry, and pharmacology. 2018;48:2337-2349.

15. Li JK, Chen C, Liu JY, Shi JZ, Liu SP, Liu B, et al. Long noncoding RNA MRCCAT1 promotes metastasis of clear cell renal cell carcinoma via inhibiting NPR3 and activating p38-MAPK signaling. Molecular cancer. 2017;16:111.

16. Kira S, Tabata K, Shirahama-Noda K, Nozoe A, Yoshimori T, Noda T. Reciprocal conversion of Gtr1 and Gtr2 nucleotide-binding states by Npr2-Npr3 inactivates TORC1 and induces autophagy. Autophagy. 2014;10:1565-1578.

17. Li S, Guo R, Peng Z, Quan B, Hu Y, Wang Y, Wang Y. NPR3, transcriptionally regulated by POU2F1, inhibits osteosarcoma cell growth through blocking the PI3K/AKT pathway. Cellular signalling. $2021 ; 86: 110074$.

18. O'Sullivan B, Brierley J, Byrd D, Bosman F, Kehoe S, Kossary C, et al. The TNM classification of malignant tumours-towards common understanding and reasonable expectations. The Lancet. Oncology. 2017;18:849-851.

19. Dooms M, Chango A, Abdel-Nour A. [Quantitative PCR (qPCR) and the guide to good practices MIQE: adapting and relevance in the clinical biology context]. Annales de biologie clinique. 2014;72:265269.

20. Kurien BT, Scofield RH. Western blotting. Methods (San Diego, Calif.). 2006;38:283-293. 
21. Camuzard O, Santucci-Darmanin S, Carle GF, Pierrefite-Carle V. Role of autophagy in osteosarcoma. Journal of bone oncology. 2019;16:100235.

22. Mulcahy Levy JM, Thorburn A. Autophagy in cancer: moving from understanding mechanism to improving therapy responses in patients. Cell death and differentiation. 2020;27:843-857.

23. Peake N, Hobbs A, Pingguan-Murphy B, Salter D, Berenbaum F, Chowdhury T. Role of C-type natriuretic peptide signalling in maintaining cartilage and bone function. Osteoarthritis and cartilage. 2014;22:1800-1807.

24. Jaubert J, Jaubert F, Martin N, Washburn LL, Lee BK, Eicher EM, Guénet J-L. Three new allelic mouse mutations that cause skeletal overgrowth involve the natriuretic peptide receptor $\mathrm{C}$ gene (Npr3). Proceedings of the National Academy of Sciences. 1999;96:10278-10283.

25. Esapa CT, Piret SE, Nesbit MA, Loh NY, Thomas G, Croucher PI, et al. Mice with an N-ethyl-Nnitrosourea (ENU) induced Tyr209Asn mutation in natriuretic peptide receptor 3 (NPR3) provide a model for kyphosis associated with activation of the MAPK signaling pathway. PLoS One. 2016;11:e0167916.

26. Boudin E, de Jong TR, Prickett TC, Lapauw B, Toye K, Van Hoof V, et al. Bi-allelic loss-of-function mutations in the NPR-C receptor result in enhanced growth and connective tissue abnormalities. The American Journal of Human Genetics. 2018;103:288-295.

27. Ruh M, Stemmler MP, Frisch I, Fuchs K, van Roey R, Kleemann J, et al. The EMT transcription factor ZEB1 blocks osteoblastic differentiation in bone development and osteosarcoma. The Journal of Pathology. 2021;254:199-211.

28. Habel N, Stefanovska B, Carène D, Patiño-Garcia A, Lecanda F, Fromigué O. CYR61 triggers osteosarcoma metastatic spreading via an IGF1Rß-dependent EMT-like process. BMC cancer. 2019;19:118.

29. Mao X, Jin Y, Feng T, Wang H, Liu D, Zhou Z, et al. Ginsenoside Rg3 inhibits the growth of osteosarcoma and attenuates metastasis through the Wnt/ $\beta$-catenin and EMT signaling pathway. Evidence-Based Complementary and Alternative Medicine. 2020;2020.

30. Stemmler MP, Eccles RL, Brabletz S, Brabletz T. Non-redundant functions of EMT transcription factors. Nature cell biology. 2019;21:102-112.

31. Babaei G, Aziz SG-G, Jaghi NZZ. EMT, cancer stem cells and autophagy; The three main axes of metastasis. Biomedicine \& Pharmacotherapy. 2021;133:110909.

32. Wu X, Tu BP. Selective regulation of autophagy by the Iml1-Npr2-Npr3 complex in the absence of nitrogen starvation. Molecular biology of the cell. 2011;22:4124-4133. 
33. Wang X, Gao Y, Yan Q, Chen W. Salicylic acid promotes autophagy via NPR3 and NPR4 in Arabidopsis senescence and innate immune response. Acta physiologiae plantarum. 2016;38:1-12.

34. Zhao G-s, Gao Z-r, Zhang Q, Tang X-f, Lv Y-f, Zhang Z-s, et al. TSSC3 promotes autophagy via inactivating the Src-mediated $\mathrm{PI} 3 \mathrm{~K} / \mathrm{Akt} / \mathrm{mTOR}$ pathway to suppress tumorigenesis and metastasis in osteosarcoma, and predicts a favorable prognosis. Journal of Experimental \& Clinical Cancer Research. 2018;37:1-17.

35. Polivka Jr J, Janku F. Molecular targets for cancer therapy in the PI3K/AKT/mTOR pathway. Pharmacology \& therapeutics. 2014;142:164-175.

36. Dienstmann R, Rodon J, Serra V, Tabernero J. Picking the point of inhibition: a comparative review of PI3K/AKT/mTOR pathway inhibitors. Molecular cancer therapeutics. 2014;13:1021-1031.

37. Zhang $\mathrm{H}$, Jiang $\mathrm{H}$, Zhang $\mathrm{H}$, Liu J, Hu X, Chen L. Anti-tumourefficacy of phellamurin in osteosarcoma cells: Involvement of the PI3K/AKT/mTOR pathway. European journal of pharmacology. 2019;858:172477.

38. Yue Z, Guan X, Chao R, Huang C, Li D, Yang P, et al. Diallyl disulfide induces apoptosis and autophagy in human osteosarcoma MG-63 cells through the PI3K/Akt/mTOR pathway. Molecules. 2019;24:2665.

39. Song R, Tian K, Wang W, Wang L. P53 suppresses cell proliferation, metastasis, and angiogenesis of osteosarcoma through inhibition of the PI3K/AKT/mTOR pathway. International Journal of Surgery. 2015;20:80-87.

40. Angulo P, Kaushik G, Subramaniam D, Dandawate P, Neville K, Chastain K, Anant S. Natural compounds targeting major cell signaling pathways: a novel paradigm for osteosarcoma therapy. Journal of hematology \& oncology. 2017;10:10.

41. Loh AHP, Stewart E, Bradley CL, Chen X, Daryani V, Stewart CF, et al. Combinatorial screening using orthotopic patient derived xenograft-expanded early phase cultures of osteosarcoma identify novel therapeutic drug combinations. Cancer letters. 2019;442:262-270.

\section{Figures}


a

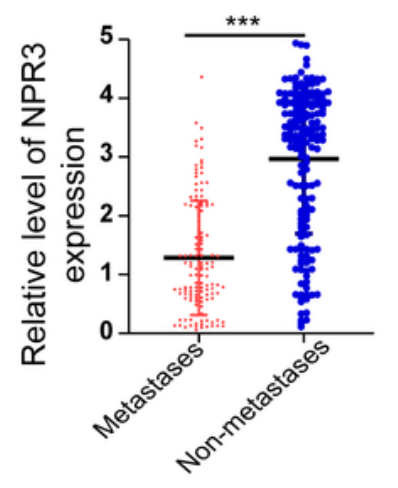

b

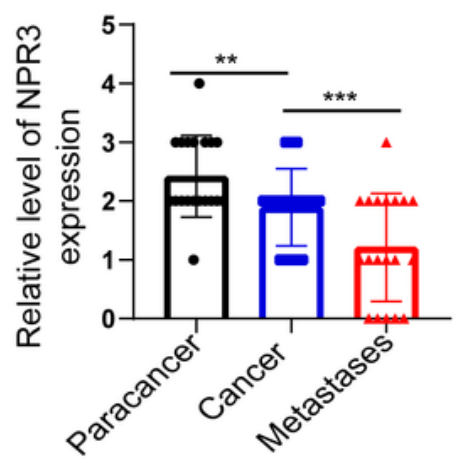

C

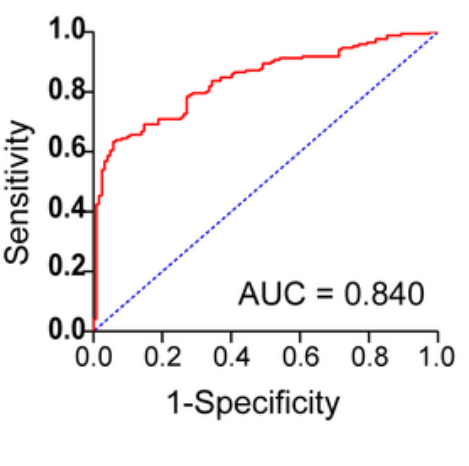

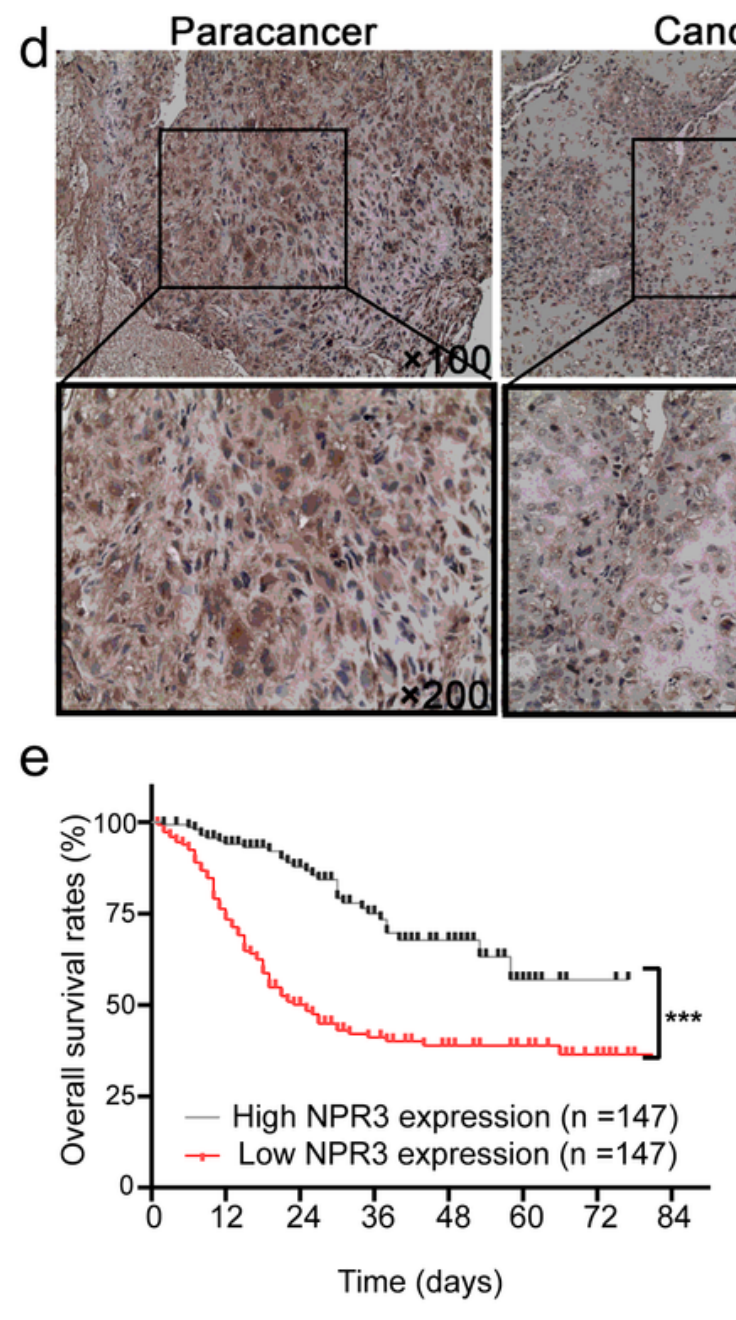

Cancer

Metastases

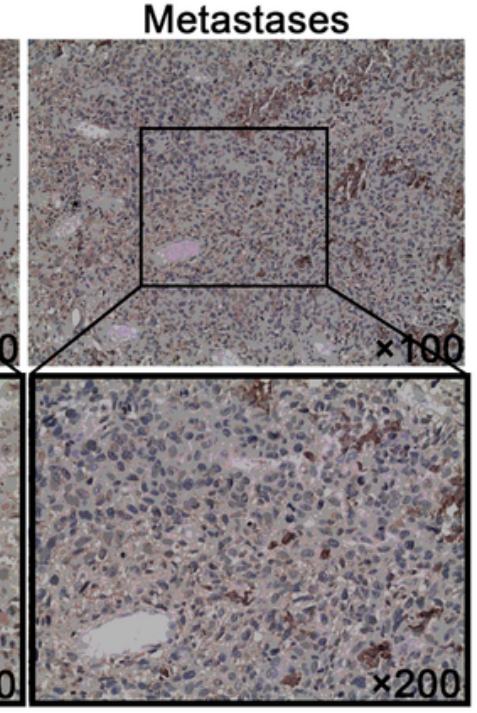

$\mathrm{f}$

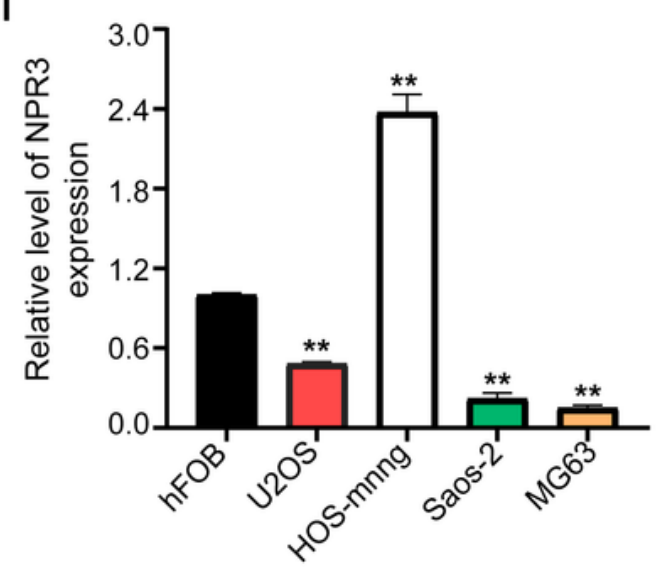

\section{Figure 1}

Downregulation expression of NPR3 is correlated with the tumour metastasis and poor prognosis in osteosarcoma patients. (a) Using qRT-PCR analysis, comparison of NPR3 mRNA expression in 172 primary osteosarcoma tissues from patients without lung metastasis, 122 primary osteosarcoma tissues from patients with lung metastasis using GAPDH as an internal loading control. (b) The IHC scores of NPR3 from 19 cases with or without metastases including the primary bone tumour, para-cancer tissue. 
Data are presented as the mean \pm standard deviation. ${ }^{\star} p<0.05$. (c) Evaluation of differential diagnosis of tumour metastases using the expression of NPR3 by ROC curve analysis. The area under the receiver operating characteristic (AUC) curve was 0.840 (0.7951-0.8844, $p<0.001)$. (d) Representative images are shown from IHC staining of NPR3 expression (Magnification at $\times 100$ and $\times 200$ ). (e) Using qRT-PCR analysis, comparison of NPR3 mRNA expression in human osteosarcoma cells (U2OS, HOS-mnng, Saos2, MG63) and one human normal bone cell hFOB using GAPDH as an internal loading control. (f) KaplanMeier survival analysis of osteosarcoma patients. OS curves for osteosarcoma patients with high NPR3 expression $(n=147)$ and low NPR3 expression $(n=147) .{ }^{\star \star \star} p<0.001$. 
a

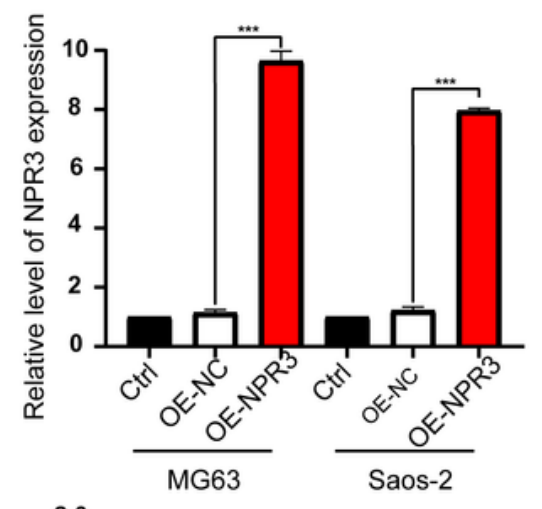

C

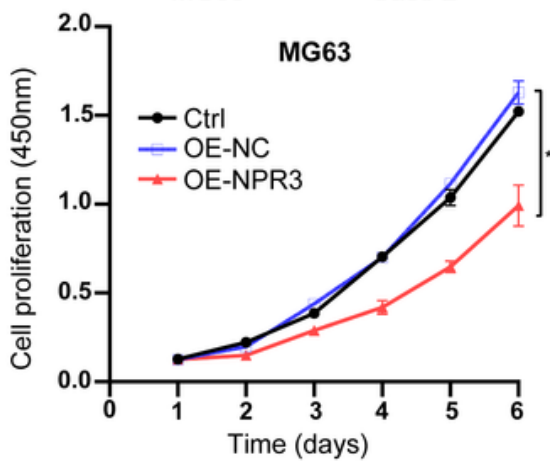

e

e Ctrl OE-NC OE-NPR3
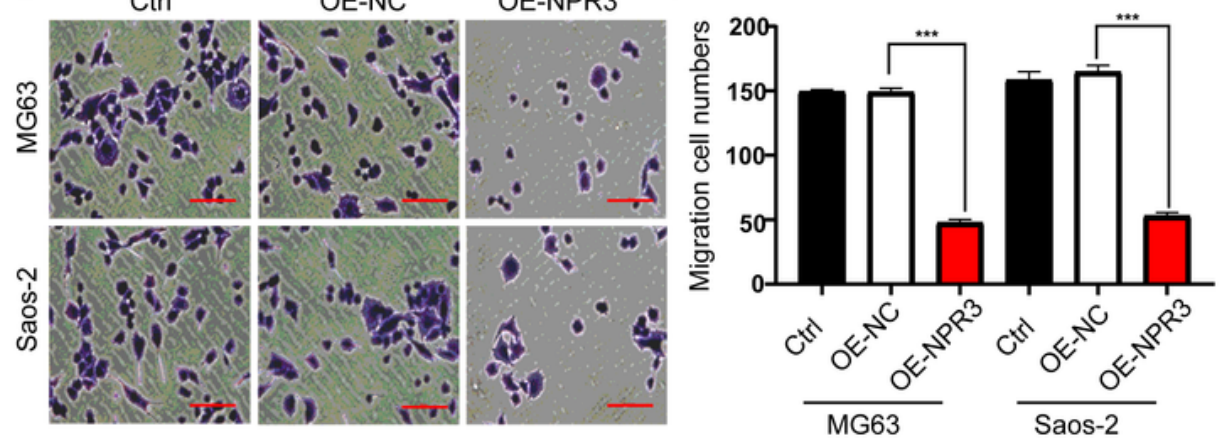

9

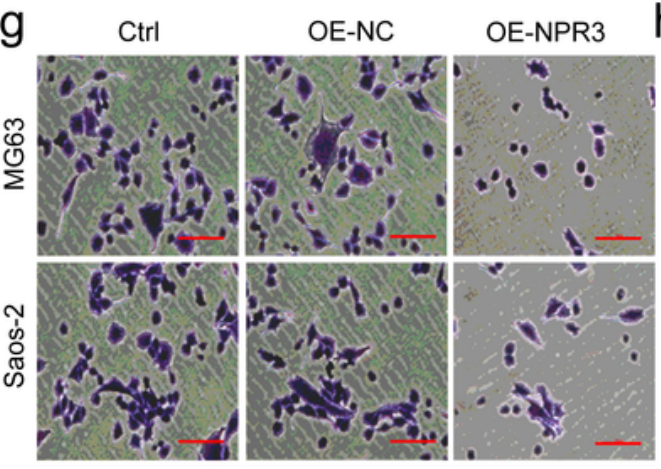

b
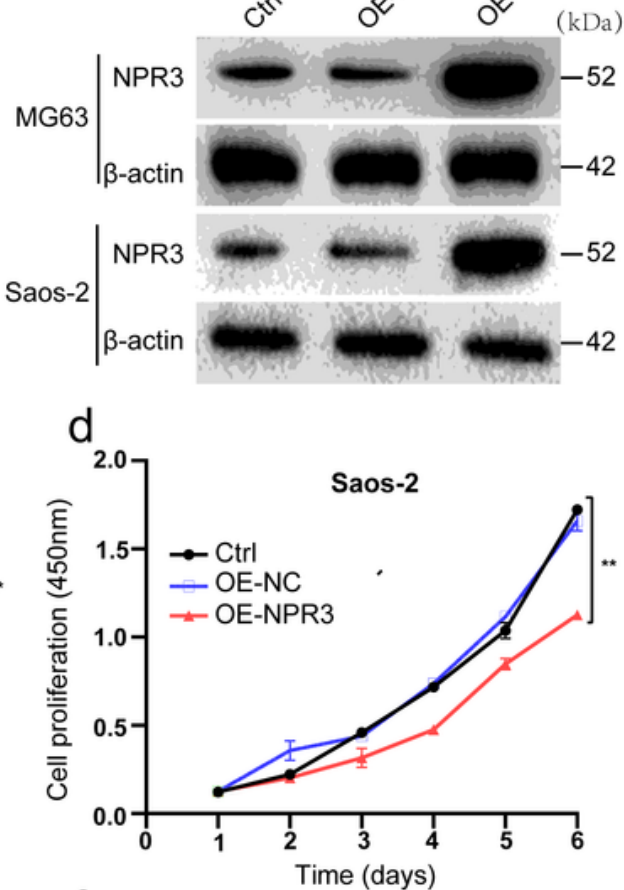

f

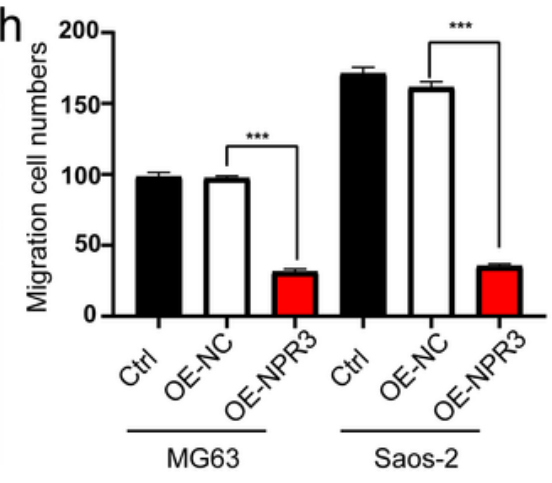

\section{Figure 2}

NPR3 overexpression inhibits growth, invasion and migration of osteosarcoma cells MG63 and Saos-2.

(a) Using qRT-PCR and (b) Western blot analysis, the NPR3 mRNA and protein expression levels increased significantly after transfection with vectors containing a constitutively OE-NPR3 or OE-NC for $48 \mathrm{~h}$ in OS cell lines MG63 and Saos-2. (c) and (d) Using CCK8 assay, cell viability was detected in the OS cells lines MG63 and Saos-2, after transfection with vectors containing a constitutively OE-NPR3 or OE- 
$\mathrm{NC}$; (e) and (f) Using a cell migration assay, migratory OS cell numbers after transfection were measured and subsequently subjected to quantitative analysis. (g) and (h) Using the invasion assay, the effects of OS cell lines MG63 and Saos-2 were measured after transfection with vectors containing constitutively OE-NPR3 or OE-NC *p $<0.05,{ }^{*} \mathrm{p}<0.01,{ }^{\star *} \mathrm{p}<0.001$ compared with the Ctrl. Data are presented as the mean \pm standard deviation from three independent experiments.
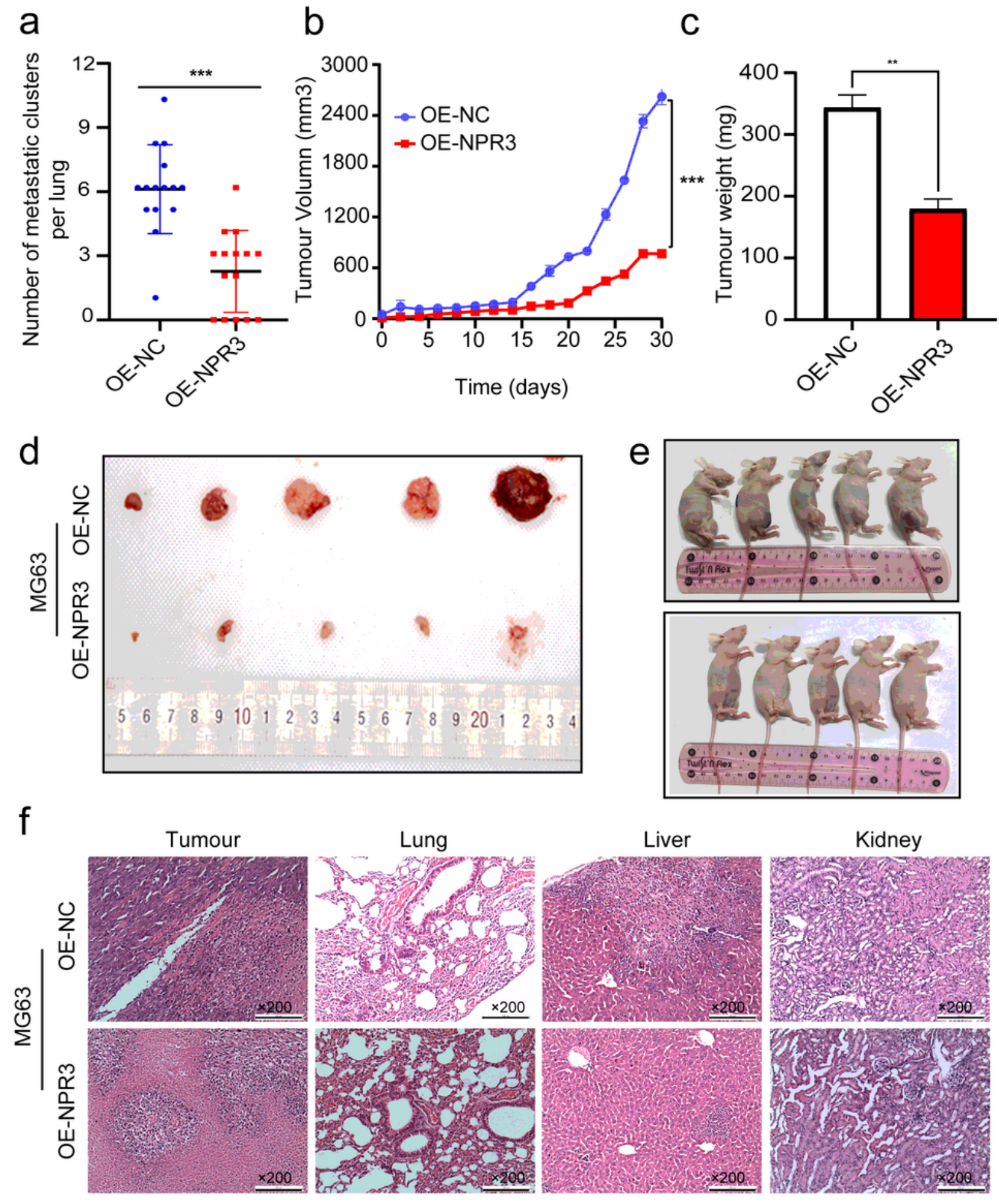

Figure 3 
Overexpression of NPR3 inhibits the tumour growth and metastasis of osteosarcoma in vivo. Stable OS cells overexpressing NPR3 were injected into nude mice. At the indicate times, (a) number of metastatic clusters per lung, (b) tumour volume, (c) primary bone tumour, (d) tumour weight were measured (mean \pm standard deviation; $n=15$ ). (e) In vivo imaging data are presented. (f) Representative metastatic foci of lungs, livers and kidney were subjected to anatomical and histological analyses. The data are shown as the mean \pm standard deviation. ${ }^{*} p<0.05,{ }^{*} \mathrm{p}<0.01,{ }^{\star *} \mathrm{p} p<0.001 \mathrm{vs}$. OE-NC.
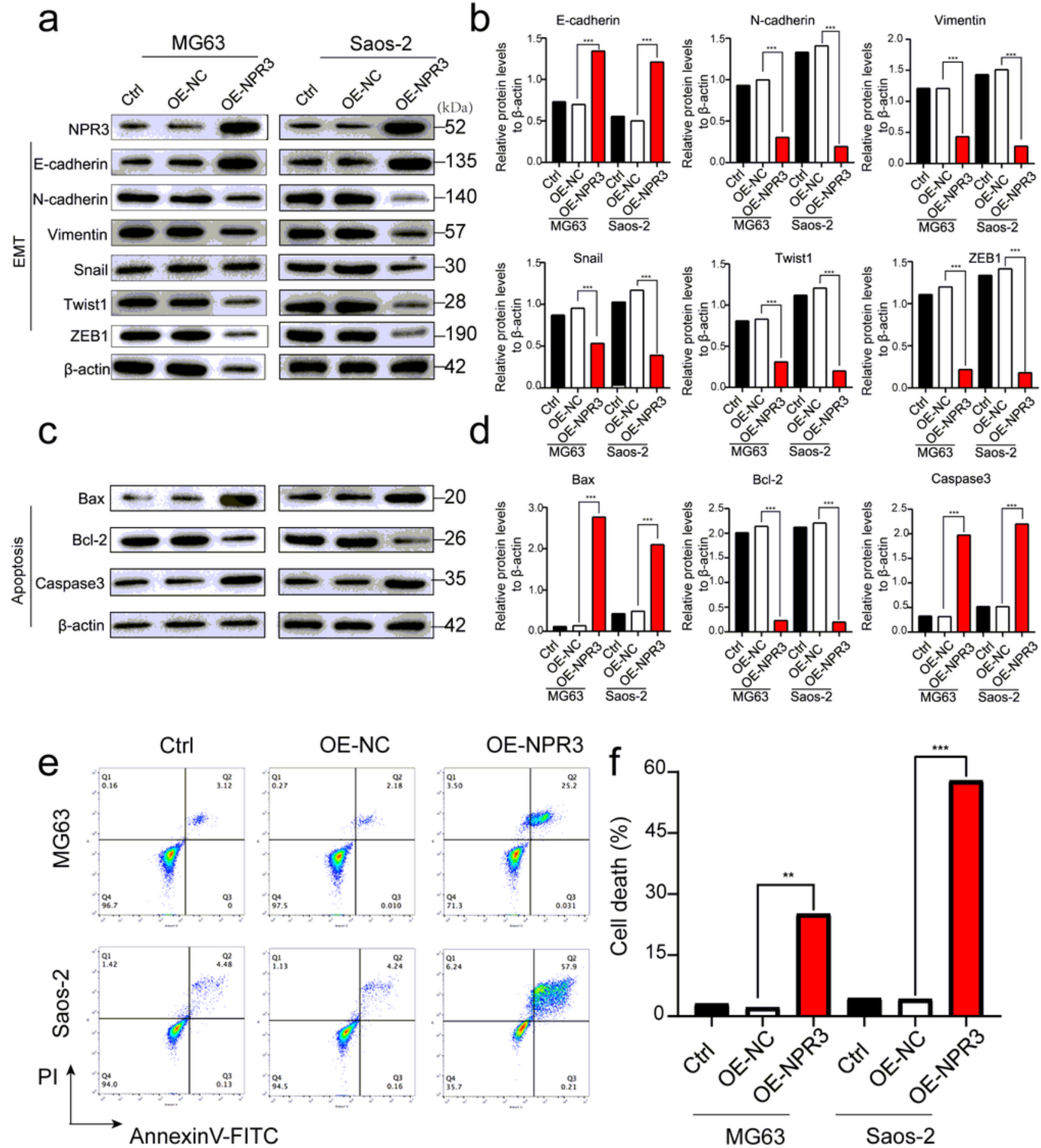

Figure 4 
Effects of NPR3 overexpression on EMT and apoptosis, which were correlated to tumour metastasis. After transfections of plamid $(a, b)$ EMT markers, including E-cadherin, N-cadherin, vimentin, Snail, Twist and ZEB1 and (c, d) apoptosis markers Bax, Bcl-2, and caspase 3 were measured by WB in Saos-2 and MG63 cells. $\beta$ actin was used as an internal control. The data are shown as the mean \pm standard deviation. ${ }^{*} p<0.05$ vs. ctrl. (e, f) Cell death of Saos-2 and MG63 cells was determined by staining with Annexin V-FITC and PI using flow cytometry.
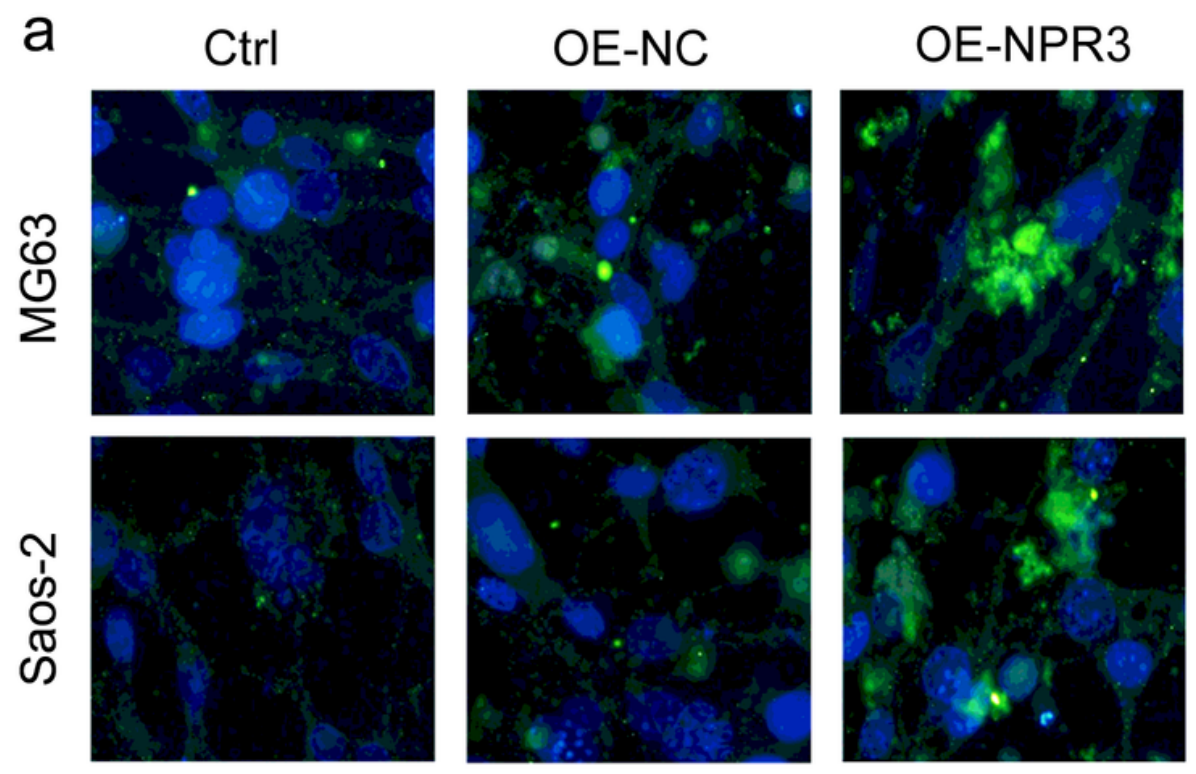

$\mathrm{b}$
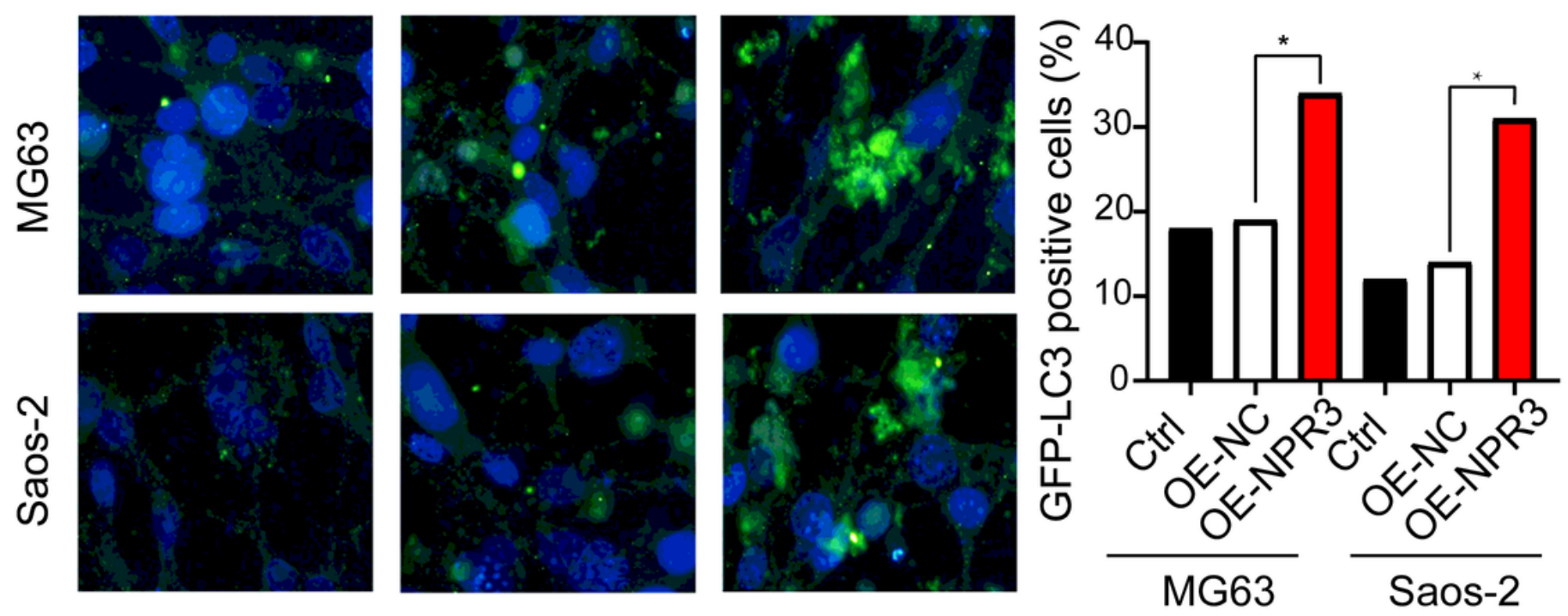

C

d

$(\mathrm{kDa})$
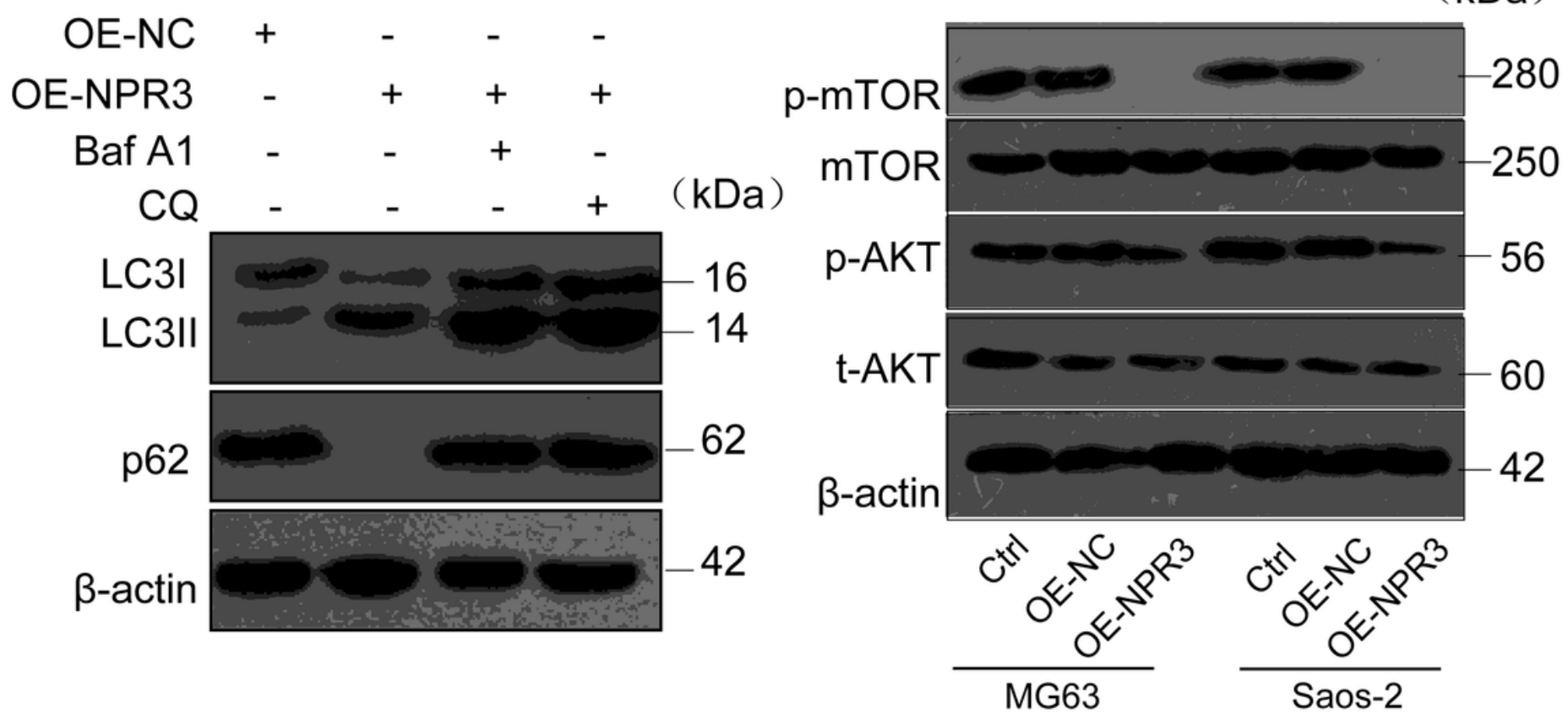

\section{Figure 5}

NPR3 overexpression promoted autophagy in OS cell lines MG63 and Saos-2. (a) Saos-2 GFP LC3/MG63 GFP LC3 cells which stably express GFP LC3 were used to detect autophagy after the cells were 
transfected with plasmid for $48 \mathrm{~h}$. (b) Percentage of GFP LC3 punctate positive cells was quantified and analyzed. (c) LC3-II and p62 expression were analyzed by WB from Saos-2 and MG63 cells transfected with OE-NC or OE-NPR3 for 48 h. Saos-2 and MG63 cells were treated with CQ or Baf-A1 for 48 hours. (d) Decreased phosphorylation levels of AKT and mTOR in OE-NPR3 OS cells were detected by WB in Saos-2 and MG63 cells. $\beta$ actin was used as an internal control. Data are presented as the mean \pm standard deviation from three independent experiments. ${ }^{*} p<0.05$ vs. ctrl.
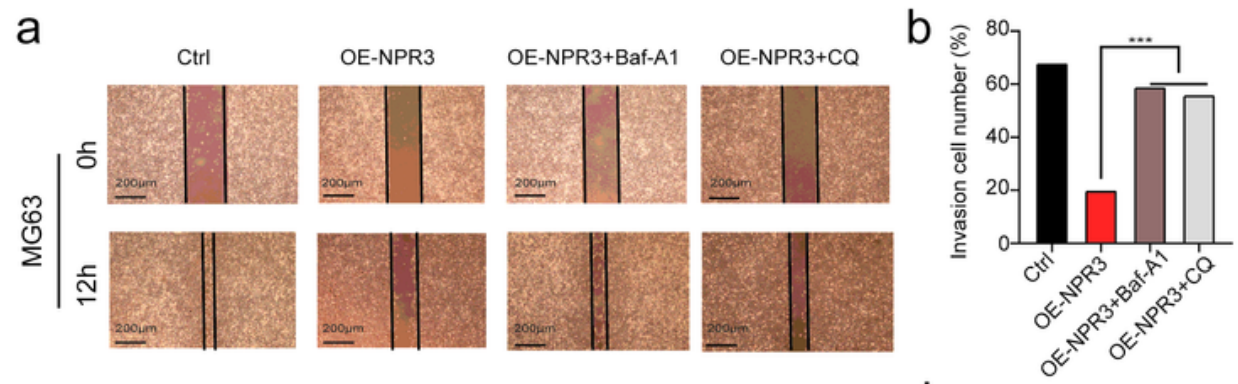

C
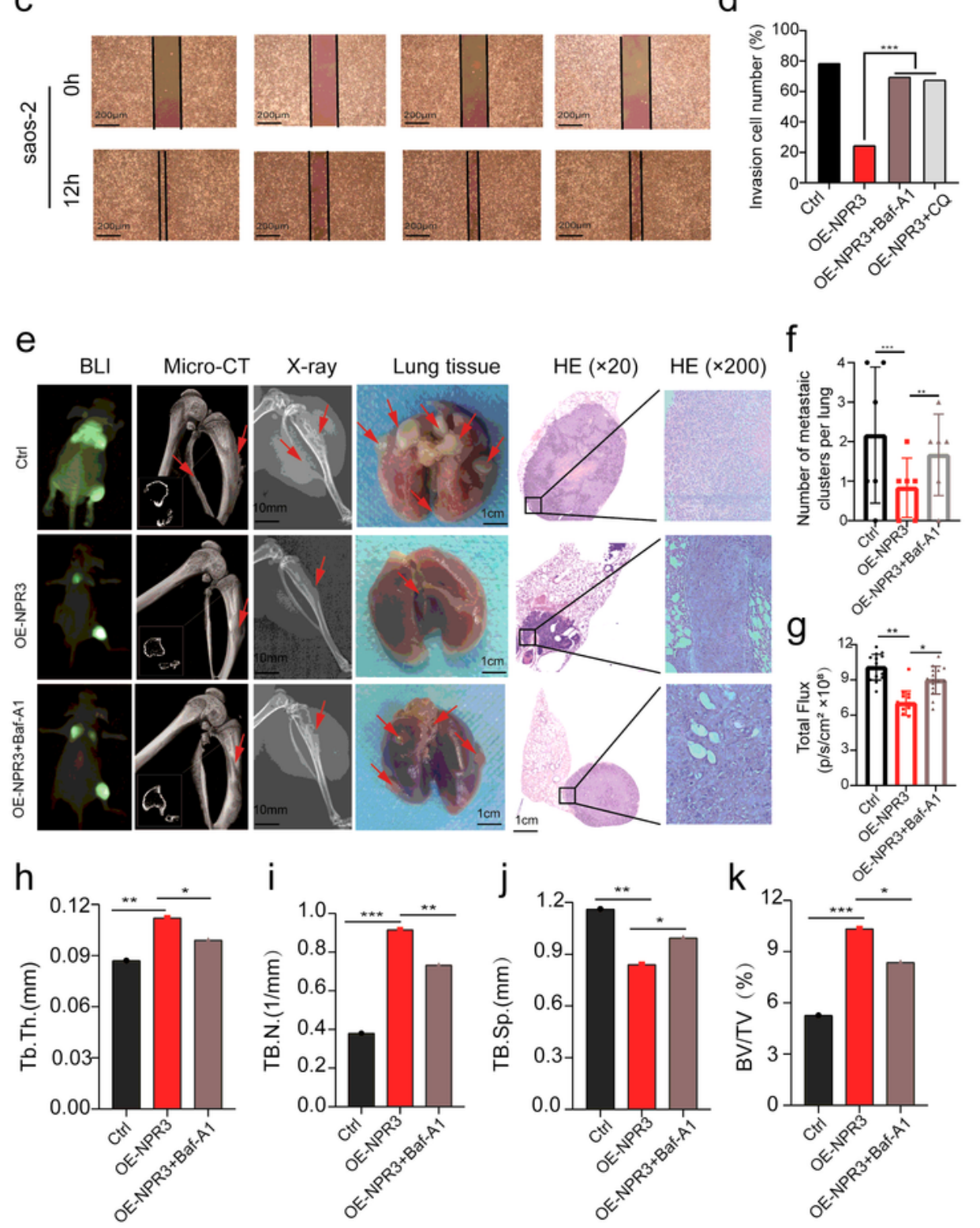

Figure 6 
Autophagy inhibitors Baf-A1 and CQ attenuated the inhibitory effect of NPR3 on tumour invasion in vitro, tumour growth and metastasis in vivo. (a) and (c) Wound healing was conducted in Saos-2 cells transfected with OE-NPR3 with or without autophagy inhibitors Baf-A1 and CQ. (b) and (d) Cell migration numbers were measured after $12 \mathrm{~h}$. Stable overexpressing NPR3 MG63 were injected into nude mice. At the indicate times, (e) pulmonary metastasis, BLI, Micro-CT assay and X-ray were used to measure the primary tumour growth and lung metastasis in nude mice deal with autophagy inhibitor Baf-A1. (f) number of metastatic clusters per lung. (g) The total flux of BLI. (h-k) Bone injury were measured (mean \pm standard deviation; $\mathrm{n}=15$ ). 
a

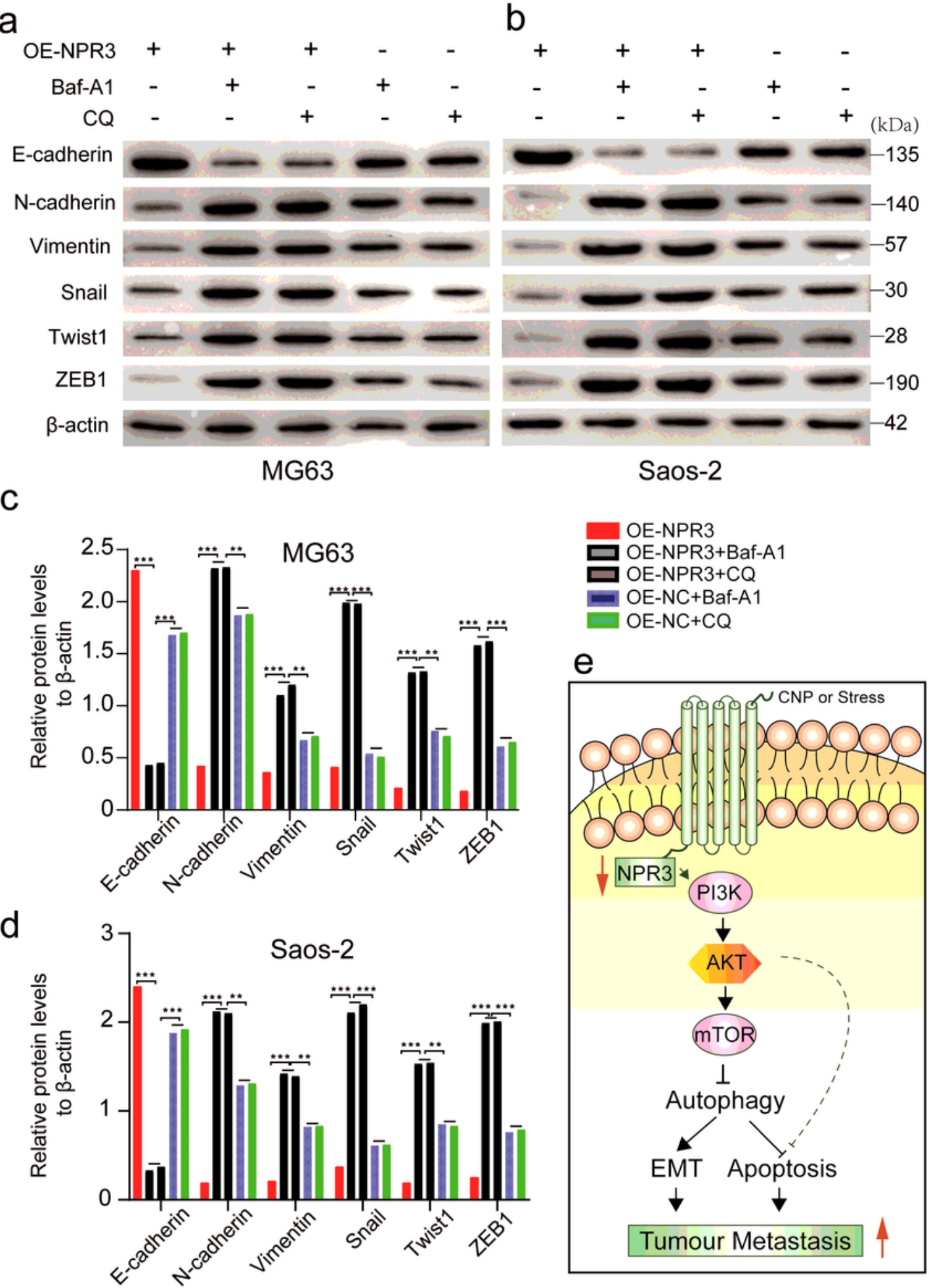

Figure 7

Autophagy inhibitors Baf-A1 attenuated the effect of NPR3 on the EMT. EMT markers, including Ecadherin, N-cadherin, vimentin, Snail, Twist and ZEB1 were measured by western blotting in osteosarcoma cells MG63 $(a, c)$ and Saos-2 (b, d). $\beta$ actin was used as an internal control. The data are shown as the mean \pm standard deviation. (e) A working model for NPR3 in the metastases of OS. 